\title{
Clinical application of a modular ankle robot for stroke rehabilitation
}

\author{
Larry W. Forrester ${ }^{\mathrm{a}, \mathrm{b}, \mathrm{c}, \mathrm{h}, *}$, Anindo Roy ${ }^{\mathrm{a}, \mathrm{c}, \mathrm{d}, \mathrm{h}}$, Ronald N. Goodman $^{\mathrm{a}, \mathrm{h}}$, Jeremy Rietschel $^{\mathrm{a}, \mathrm{h}}$, \\ Joseph E. Barton ${ }^{\mathrm{a}, \mathrm{c}, \mathrm{h}}$, Hermano Igo Krebs ${ }^{\mathrm{c}, \mathrm{e}}$ and Richard F. Macko ${ }^{\mathrm{a}, \mathrm{b}, \mathrm{c}, \mathrm{f}, \mathrm{g}, \mathrm{h}}$ \\ ${ }^{\mathrm{a}}$ VA RR\&D Maryland Exercise and Robotics Center of Excellence, Baltimore, MD, USA \\ ${ }^{\mathrm{b}}$ Department of Physical Therapy and Rehabilitation Science, University of Maryland School of Medicine, \\ Baltimore, MD, USA \\ ${ }^{\mathrm{c}}$ Department of Neurology, University of Maryland School of Medicine, Baltimore, MD, USA \\ ${ }^{\mathrm{d}}$ Department of Bioengineering, University of Maryland School of Engineering, College Park, MD, USA \\ ${ }^{\mathrm{e}}$ Department of Mechanical Engineering, Massachusetts Institute of Technology, Cambridge, MA, USA \\ ${ }^{\mathrm{f}}$ Department of Medicine, University of Maryland School of Medicine, Baltimore, MD, USA \\ ${ }^{\mathrm{g}}$ Geriatrics Research, Education and Clinical Center, Baltimore Veterans Affairs Medical Center, Baltimore, \\ $M D, U S A$ \\ ${ }^{\mathrm{h}}$ University of Maryland Rehabilitation and Orthopedics Institute (UMROI), University of Maryland School of \\ Medicine, Baltimore, MD, USA
}

\begin{abstract}
.
BACKGROUND: Advances in our understanding of neuroplasticity and motor learning post-stroke are now being leveraged with the use of robotics technology to enhance physical rehabilitation strategies. Major advances have been made with upper extremity robotics, which have been tested for efficacy in multi-site trials across the subacute and chronic phases of stroke. In contrast, use of lower extremity robotics to promote locomotor re-learning has been more recent and presents unique challenges by virtue of the complex multi-segmental mechanics of gait.

OBJECTIVES: Here we review a programmatic effort to develop and apply the concept of joint-specific modular robotics to the paretic ankle as a means to improve underlying impairments in distal motor control that may have a significant impact on gait biomechanics and balance.

METHODS: An impedance controlled ankle robot module (anklebot) is described as a platform to test the idea that a modular approach can be used to modify training and measure the time profile of treatment response.

RESULTS: Pilot studies using seated visuomotor anklebot training with chronic patients are reviewed, along with results from initial efforts to evaluate the anklebot's utility as a clinical tool for assessing intrinsic ankle stiffness. The review includes a brief discussion of future directions for using the seated anklebot training in the earliest phases of sub-acute therapy, and to incorporate neurophysiological measures of cerebro-cortical activity as a means to reveal underlying mechanistic processes of motor learning and brain plasticity associated with robotic training.

CONCLUSIONS: Finally we conclude with an initial control systems strategy for utilizing the anklebot as a gait training tool that includes integrating an Internal Model-based adaptive controller to both accommodate individual deficit severities and adapt to changes in patient performance.
\end{abstract}

Keywords: Stroke, rehabilitation robotics, hemiparetic gait, lower extremity robotics, motor learning

\section{Introduction}

Gait and balance deficits limit functional mobility

*Address for correspondence: Larry W. Forrester, 100 Penn Street, Suite 115, Baltimore, MD 21201, USA. Tel.: +1410 706 5212;

E-mail: LForrester@som.umaryland.edu. and contribute to more than $70 \%$ of stroke survivors sustaining a fall within six months (Forster, \& Young, 1995), leading to higher risks for hip and wrist fractures 
in the first year (Dennis, Lo, McDowell, \& West, 2002; Kanis, Oden, \& Johnell, 2001; Ramenemark, Nyberg, Borssen, \& Gustafson, 1998). These disabling consequences of stroke not only limit participation in community life but also set the stage for a sedentary lifestyle that reinforces learned nonuse and can lead to further declines in mobility and balance functions. Advances in our understanding of neuroplasticity and motor learning post-stroke are now being leveraged with the use of robotics technology to change the natural history of stroke recovery. In terms of clinical translation, the greatest advances have been made with the use of upper extremity (UE) robotics, which have been tested for efficacy in multi-site trials (Burgar et al. 2011; Lo et al. 2011). In contrast, the implementation of lower extremity (LE) robotics to promote locomotor re-learning through massed practice has been more recent and presents unique challenges by virtue of the complex dynamics of gait, including the coordination of both legs and the multi-segmental balance control inherent to upright locomotion.

Early applications of robotics to UE therapy were chiefly directed at whole arm movements that are largely defined by end effector trajectories, as when reaching with the entire shoulder-elbow-wrist complex to contact a target (Krebs, Hogan, Aisen, \& Volpe, 1998; Krebs et al., 2007; Lum, Burgar, Kenney, \& Van der Loos, 1999; Reinkensmeyer, Dewald, \& Rymer, 1999). More recently a multi-modular approach has been advocated to apply therapy at the individual joints to address specific deficits or to promote a sequential approach (Krebs, Volpe, Lynch, \& Hogan, 2005). Development of LE robotics has followed a similar pattern. The first large scale efforts were aimed at re-creating the essentials of task-oriented treadmill training with partial body weight support (PBWS), emphasizing the consistency of gait-like stepping patterns that could be repeated for long periods without relying on therapists to assist with the stepping actions (Colombo, Joerg, Schreier, \& Dietz, 2000; Hesse \& Uhlenbrock, 2000; Schmidt, Sorowaka, Hesse, \& Bernhardt, 2003). More recently we have moved into modular LE robotics, using impedance control to better understand and optimize motor learning and customize these approaches to deficit profiles and clinical setting (Forrester, Roy, Krebs, \& Macko, 2011; Forrester, Roy, Krywonis, Kehs, Krebs, \& Macko, 2012; Roy, Forrester, \& Macko, 2011; Roy, Krebs, Bever, Forrester, Macko, \& Hogan, 2011). In concert with these engineering advances are studies of cortical neurophysiology that may provide insights into the mechanisms of activity dependent plasticity and their optimal delivery to mediate motor learning and improve functional outcomes (Goodman, Macko, Roy, Forrester, 2012; Halsband, \& Lange, 2006).

This review starts with a brief background on the evolution of contemporary interventions aimed at improving gait and mobility functions after hemiparetic stroke. We begin with earlier PWBS treadmill approaches that fostered the emergence of robotic exoskeletons and other gait trainers that are designed to facilitate whole body locomotor training using preprogrammed walking patterns. We then shift to the idea that robotic modules designed for specific LE joints may offer another avenue to promote improved walking function. Here we describe the impedance controlled ankle robot module (anklebot) that has provided a platform to test this notion, including the device's intrinsic measurement and actuation capabilities. Pilot results are presented from initial studies of seated visuomotor anklebot training during the chronic and sub-acute phases post-stroke. The seated approach is also shown to be viable for using electroencephalography (EEG) to characterize neural mechanisms of ankle motor control. Finally, we present a conceptual framework for an adaptive controller that integrates real-time performance to both accommodate individual deficit severities and adapt to changes in patient performance.

\section{Treadmill locomotor training}

As prelude to LE robotics, the advent of treadmill training for gait rehabilitation after stroke was largely predicated on results from various spinalized cat models that showed locomotor patterning could be elicited without supraspinal inputs to the fore- and hindlimbs (Grillner, 1975; Grillner, \& Wallen, 1985). Pioneering studies in persons with spinal cord injuries and stroke sought to test whether humans might benefit from treadmill as a stimulus for gait patterning and did show that it was possible to train severely impaired patients with varying degrees of PBWS (Barbeau, \& Visintin, 2003; Hassid, Rose, Commisarow, Guttry, \& Dobkin, 1997; Hesse et al., 1995; Hesse, Bertelt, Schaffrin, Malezic, \& Mauritz, 1994). Evidence of improved walking function was observed, and efforts to discern whether the treadmill approach yields meaningful gains in function are ongoing. A variant of the PBWS treadmill method has been to focus less on gait patterning and assisted stepping and more on the aerobic exercise aspect of effortful walking. In chronic stroke this 
approach provides a locomotor stimulus with massed practice and has shown marked improvements in cardiovascular fitness and floor walking velocity, along with fMRI changes in paretic leg activation (Luft et al., 2008; Macko et al., 2005). These findings provide evidence that, even years after a disabling stroke, locomotor improvements with treadmill training activate bilateral mesencephalic, cerebellar, and ipsilesional cortex areas, suggesting mechanisms of neuroplasticity. Other promising approaches include the use of splitbelt treadmill, which aims to induce motor adaptations that carry over to more symmetrical stepping patterns when transferred to over ground walking (Reisman et al., 2007; Reisman, Wityk, Silver, \& Bastian, 2009). Still, there remain questions about the impact of treadmill compared to more generic locomotor activities such as home walking and exercise programs (Duncan, Sullivan, Behrman et al., 2011), which appear just as effective for mediating improvements in independent mobility outcomes. Moreover, the issues of whether these approaches improve the biomechanical quality of gait and dynamic balance are unsettled.

\section{Lower extremity robotics for locomotor training}

The promise of using the driving stimulus of the treadmill to train locomotor patterning motivated major innovations in the area of powered gait orthoses and other robotic gait trainers. The Lokomat is one of the first widely used LE robots, designed as a bilateral computerized gait orthosis used in conjunction with PBWS during treadmill walking (Colombo, Joerg, Schreier, \& Dietz, 2000). This patterned locomotor training has produced positive effects on self-selected gait speed, but has not shown an advantage compared to equal dose-intensity of standard physical therapy (Hornby, Campbell, Kahn, Demott, Moore, \& Roth, 2008; Westlake, \& Patten, 2009). The foot-plate activated Gait Trainer is another device that evokes a physiological stepping pattern by moving the feet symmetrically as the patient stands on two moving foot supports (Hesse, \& Uhlenbrock, 2000; Schmidt, Sorowka, Hesse, \& Bernhardt, 2003). This too has shown benefits, but the absence of a true swing phase and ground impact may be limitations when transferring to over ground walking (Schmidt, Werner, Bernhardt, Hesse, \& Kruger, 2007). Another innovation has been to elaborate on the treadmill approach by introducing robotic actuators that interface with the pelvic girdle to promote weight shifts and multi-planar rotations that affect stepping as the patient walks (Ichinose et al., 2003). As highlighted in a Cochrane review, there are potential benefits from using robotics in gait training after stroke, but we have much to learn about specific interventions (Mehrholz, Werner, Kugler, \& Pohl, 2007). Among the unknowns is whether targeting specific joint deficits will impact whole task functions such as gait and balance. An example of this is the Rutgers ankle system that employs a seated training approach to engage the paretic ankle in performing virtual reality (VR) exercises to improve motor control (Mirelman, Bonato, \& Deutsch, 2009), which carries over into faster floor walking and increased walking activity at home. Beyond this example, few studies have investigated modular robotics as a way to improve joint motor control and its impact on gait and balance.

\section{Development and preclinical testing of the anklebot}

Over the past few years our VA Center of Excellence has investigated the potential for using a modular ankle robot ("anklebot") that translates upper extremity (UE) impedance-controlled robotics technology to gait rehabilitation in stroke. The paretic ankle was targeted because it is the site of major biomechanical contributions to normal gait (Robertson, \& Winter, 1980) and to the sensorimotor control of balance (Nashner, 1977). In particular, the generation of mechanical power through the paretic ankle is severely impaired after stroke (Olney, Griffin, Monga, \& McBride, 1991). Reduced propulsive impulses during the paretic stance phase of hemiparetic gait may also reflect this ankle power deficit and contribute to interlimb asymmetry (Bowden, Balasubramanian, Neptune, and Kautz, 2006). Important for gait safety after stroke are ankle dorsiflexion, eversion deficits and spasticity that contribute to foot drop and falls. These deficits are clinically addressed with use of ankle-foot orthoses, which reinforce a pattern of non-use. Improving paretic ankle contributions to the biomechanics of walking could potentially benefit gait velocity, reduce fall risk, and reduce increased demands at the nonparetic limb, making it a logical focus for robotic intervention.

The anklebot allows ankle movement in all three degrees of freedom (DOF) but it actuates only two of those, i.e., dorsi-plantar flexion (DF/PF) and inversion-eversion (INV-EV) via linear actuators mounted in parallel. It is underactuated i.e., actuated in fewer DOFs than are anatomically present, because 

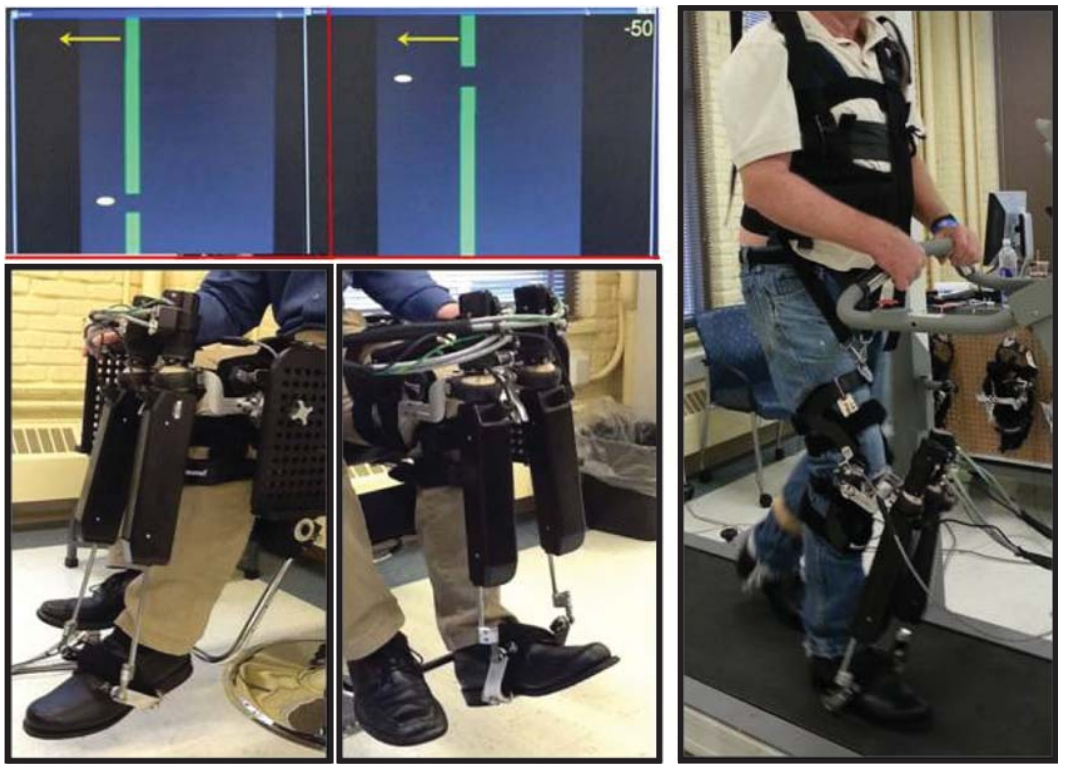

Fig. 1. The modular 2-DOF actuated and impedance-controlled anklebot is designed for use in seated position with video feedback (left panel plantar flexion and center panel - dorsiflexion) and for upright walking (right panel).

internal-external rotation is limited at the ankle with the orientation of the foot in the transverse plane being controlled primarily by rotation of the leg. Moreover, underactuation allows the device to be installed without requiring precise alignment with the patient's joint axes (ankle and subtalar joints). The anklebot is designed to operate in multiple therapeutic settings, including in seated or recumbent positions, and walking on a treadmill or over ground (Fig. 1). The robot is interfaced with computer games "played" by moving the ankle in DF/PF and/or INV/EV ranges. It attaches proximally to a knee brace and distally at a shoe-mounted plate with two actuators to deliver torques as required in both ranges. A customized fitting is used to maximize comfort and protect from potential skin irritations. The proximal attachment site is mounted anterior to an orthopedic knee brace that is lined with foam pads and has cushioned straps.

In order to quantify the accuracy of anklebot's estimation of ankle angles, the device was validated by comparison to independent external measurements (Roy et al., 2009). Briefly, the mean positional error across full range of movement is $\leq 1^{\circ}$ in both DOFs (maximum $1.5^{\circ}$ ) and the error in torque estimation is $<1 \mathrm{Nm}$. The inherent static friction of the device is low $(\sim 3 \mathrm{~N})$ that results in $<0.75 \mathrm{Nm}$ of stiction torque. Another feature is the ability to record and/or program a movement trajectory and generate necessary torques to reproduce the kinematics; this may be useful as a means to guide the ankle-foot system through a prescribed pattern. Hence the intrinsic kinematic and kinetic measurements of the anklebot are valid and highly reliable. From recording these basic data it becomes possible to derive a number of performance metrics that provide a perspective on the underlying movement control. Specifically the angular position time series in the $\mathrm{DF} / \mathrm{PF}$ and/or INV/EV ranges $(200 \mathrm{~Hz})$ can be exported to quantify the peak displacements in each direction and further processed to obtain the first derivatives for the mean and peak velocities. Jerk, the third derivative of the positional data, is normalized to peak velocity as a measure of movement smoothness. Additional performance data are available from the records of task success (e.g., moving the robot to hit screen targets), active ranges of motion (AROM), and estimates of peak isometric torques.

\section{Seated visuomotor anklebot training}

A fundamental conceptual question in stroke neuromotor rehabilitation is whether to emphasize taskspecific gait pattern training, or modular and joint specific mass training aimed at specific stroke impairments. A 6 -week program $(3 \times$ weekly $=18$ sessions $)$ of a seated performance-based robot training was 
conducted to determine initial feasibility for using the ankle robot in extended training in individuals with chronic stroke (Forrester, Roy, Krebs, \& Macko, 2011). Subjects $(n=8 ; 62.4 \pm 10.4$ yrs.) with chronic stroke (73 \pm 37 mos. status-post), had completed conventional therapy, and had persistent LE hemiparesis with at least minimal ankle activation in both DF and PF directions.

A performance-based training protocol was implemented by playing a "racer" videogame, adapted from the MIT Manus protocols, that requires repetitive DF and $\mathrm{PF}$ of the paretic ankle to move a screen cursor "up or down" in order to pass through "gates" that approached across the screen at different vertical levels. Gate locations were individualized for each subject based on paretic ankle AROM, and level of assistance was set initially to facilitate an $80 \%$ success rate (Boyce, Coker, \& Bunker, 2006). The level of robotic support was reduced every 2 blocks (160 movements), from 125 to 75 to $25 \mathrm{Nm} / \mathrm{rad}$, increasing the volitional movement demands on the paretic ankle. Sessions also included unassisted trials before and after training, bringing the total targeted movements to 560 per day to fit within a one hour training session, including rest intervals between blocks of trials.

Improved paretic ankle motor control was indicated by changes at 6 weeks in several metrics of performance and quality of movement trajectories during volitional ankle movements in unassisted trials (Table 1). Subjects

Table 1

Motor control variables $(n=8)$ from unassisted robotic tests (Mean \pm SE)

\begin{tabular}{lrrrr}
\hline Variable (units) & Baseline & 6-weeks & $\begin{array}{c}\%- \\
\text { change }\end{array}$ & $\begin{array}{c}p \text { - } \\
\text { value }\end{array}$ \\
\hline Successful gates $(\#)$ & $20.5 \pm 3.3$ & $34.3 \pm 1.9$ & 67 & 0.003 \\
Mean velocity $(\% / \mathrm{sec})$ & $4.8 \pm 0.5$ & $9.9 \pm 1.7$ & 106 & 0.008 \\
Peak velocity $(\% / \mathrm{sec})$ & $41.4 \pm 3.0$ & $48.2 \pm 3.9$ & 16 & 0.032 \\
Mean jerk $\left(\% / \mathrm{sec}^{3}\right)$ & $302.7 \pm 18.8$ & $209.4 \pm 20.5$ & -31 & 0.032 \\
\hline
\end{tabular}

Table 2

Spatio-temporal gait parameters $(n=8)$ pre-post 6-weeks ankle robot training (Mean $\pm \mathrm{SE}$ )

\begin{tabular}{lllrr}
\hline Variable (units) & Baseline & 6-weeks & $\begin{array}{c}\% \text { - } \\
\text { change }\end{array}$ & $\begin{array}{c}p \text { - } \\
\text { value }\end{array}$ \\
\hline Walking velocity (cm/s) & $51.4 \pm 11.1$ & $61.7 \pm 10.9$ & 20 & 0.032 \\
Stride length (cm) & $78.2 \pm 10.5$ & $86.3 \pm 9.3$ & 10 & 0.048 \\
Cadence (steps/min) & $75.3 \pm 7.5$ & $83.4 \pm 8.1$ & 11 & 0.045 \\
NP-step length (cm) & $37.5 \pm 5.7$ & $41.8 \pm 5.2$ & 11 & 0.032 \\
P-step length (cm) & $40.7 \pm 5.3$ & $43.8 \pm 4.7$ & 8 & 0.203 \\
P-Single support (\%) & $21.1 \pm 2.4$ & $24.2 \pm 2.4$ & 15 & 0.033 \\
Double support (\%) & $46.6 \pm 4.6$ & $40.3 \pm 4.0$ & -14 & 0.010 \\
\hline
\end{tabular}

NP: Nonparetic; P: paretic. increased self-selected walking velocity, with increased stride lengths and cadence. Decreased \% double support and increased paretic \% single support suggested greater contributions from the paretic limb in control of dynamic balance. The step length increased significantly on the nonparetic side, suggesting greater forward propulsion from the paretic side. Key gait outcomes are reported in Table 2.

In summary, six weeks seated anklebot training significantly improved all robotics measured parameters of motor control and selected spatiotemporal gait parameters in chronic hemiparetic patients that had already completed all conventional rehabilitation options.

\section{Short-term motor learning}

A secondary investigation examined whether the anklebot can be utilized as a high-throughput model to assess short-term motor learning at the paretic ankle (Roy, Forrester, \& Macko, 2011). A double-arm pilot study was conducted with these same chronic stroke (ST) survivors and an equal number of age- and gendermatched healthy (HC) subjects to determine the effects of a single session of seated anklebot training. Training consisted of playing the racer videogame in DF/PF ranges as described above, followed by a re-test of an unassisted trial 48 hours later. Assessments included measures of paretic ankle motor control before and after training, and at 48 hours.

In the subjects with stroke, improved paretic ankle motor control was seen across a single training session as indexed by increased targeting accuracy, higher angular speeds, and smoother movements (Fig. 2-left panel). In contrast, healthy subjects did not make statistically significant gains in any metric after training except in the number of successful passage. Short-term motor learning was suggested by the fact that performance gains were retained at 48 hours as evidenced by the lack of statistically significant differences between the two time points (Roy, Forrester, \& Macko, 2011). These results also suggested that the anklebot may be a flexible motor learning platform with potential to detect rapid changes in motor performance post-stroke during initial testing. This sensitivity may help customize training parameters across varied patient deficit levels and stratify patients as potential responders vs. nonresponders. These findings demonstrated the possibility that in chronic hemiparetic stroke patients, anklebot can accurately modulate and measure short-term adaptation and motor learning. 

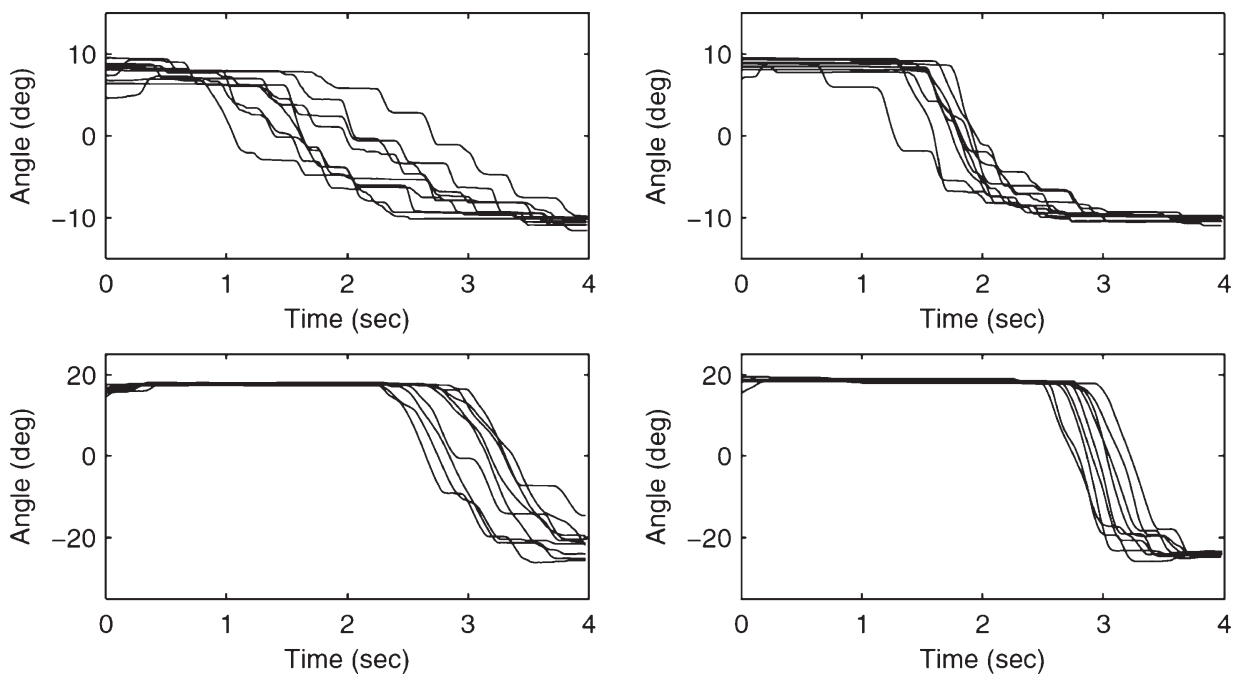

Fig. 2. Exemplar point-to-point unassisted movements made by a typical (upper) stroke and (lower) healthy subject before (left) and after training (right). (Adapted from Roy et al., J Rehabil Res Dev 2011).

\section{Assessments of passive ankle stiffness}

From the outset the anklebot was designed to help characterize LE impairments. Specifically, the device was used to estimate passive ankle stiffness (PAS) of the paretic leg as compared to older age-matched individuals and, subsequently, to evaluate how robotic training may alter this basic characteristic in the paretic ankle. An accurate estimate of PAS is potentially valuable for locomotor rehabilitation as it may provide a quantitative measure of recovery in ankle impairment resulting from an intervention. PAS was evaluated both in sagittal and in frontal planes by applying very slow perturbations $\left(5^{\circ} / \mathrm{s}\right)$ to the ankle joint over the entire range of motion with subjects in a relaxed state, as confirmed by surface electromyography (Roy, Krebs, Bever, Forrester, Macko, \& Hogan, 2011). The results showed that PAS of the paretic ankle was: a) significantly higher in subjects with chronic stroke than in healthy older subjects in three (DF, INV, and EVR) of the four ranges tested, with indistinguishable stiffness values in PF direction (Fig. 3), and b) anisotropic i.e., greater in one versus the opposite direction within a given plane of movement.

In terms of clinical relevance, there is evidence to suggest that the anisotropic nature of PAS may be attributable to the summed physiological cross-sectional area of the antagonist muscle group lengthened during passive stretch. Training-induced changes in PAS may arise from: a) altered antagonist muscle physiology, or b) distorted neurophysiology, e.g., neural factors including abnormal muscle activation and/or abnormal neural feedback. Again from a clinical perspective, this method would be easy to implement and require only a few minutes to obtain PAS estimates in multiple ranges of motion.

A secondary analysis of the six-week seated anklebot training was performed to assess potential changes in paretic PAS and to determine if those changes were related to selected aspects of walking function. PAS was measured in both the trained sagittal and untrained frontal planes at baseline and at the completion of the 18 training sessions. There were three main findings. First, anklebot training positively affected PAS in the sagittal plane (DF-PF), but not in the frontal plane. At baseline, the sagittal plane PAS was anisotropic, with significantly greater stiffness in DF than in PF; however, this was not the case in the frontal plane. After 6 weeks of training, the PAS decreased in all four directions (DF, PF, INV and EV) but statistically significant changes were observed only in the sagittal PAS-i.e., DF and PF, and in one of those directionsi.e., DF, the PAS values reverted into the ranges of age-matched controls (Fig. 4). Second, the decreases in PF PAS had a strong and significant relationship with gains in selected spatio-temporal parameters of paretic step $(\rho=-0.88)$ and stride $(\rho=-0.82)$ lengths. Since the PAS contributes to the total mechanical impedance of the ankle joint, the changes in PF PAS may have enabled subjects to position their foot more efficiently. Third, positive gains in paretic step length and single 

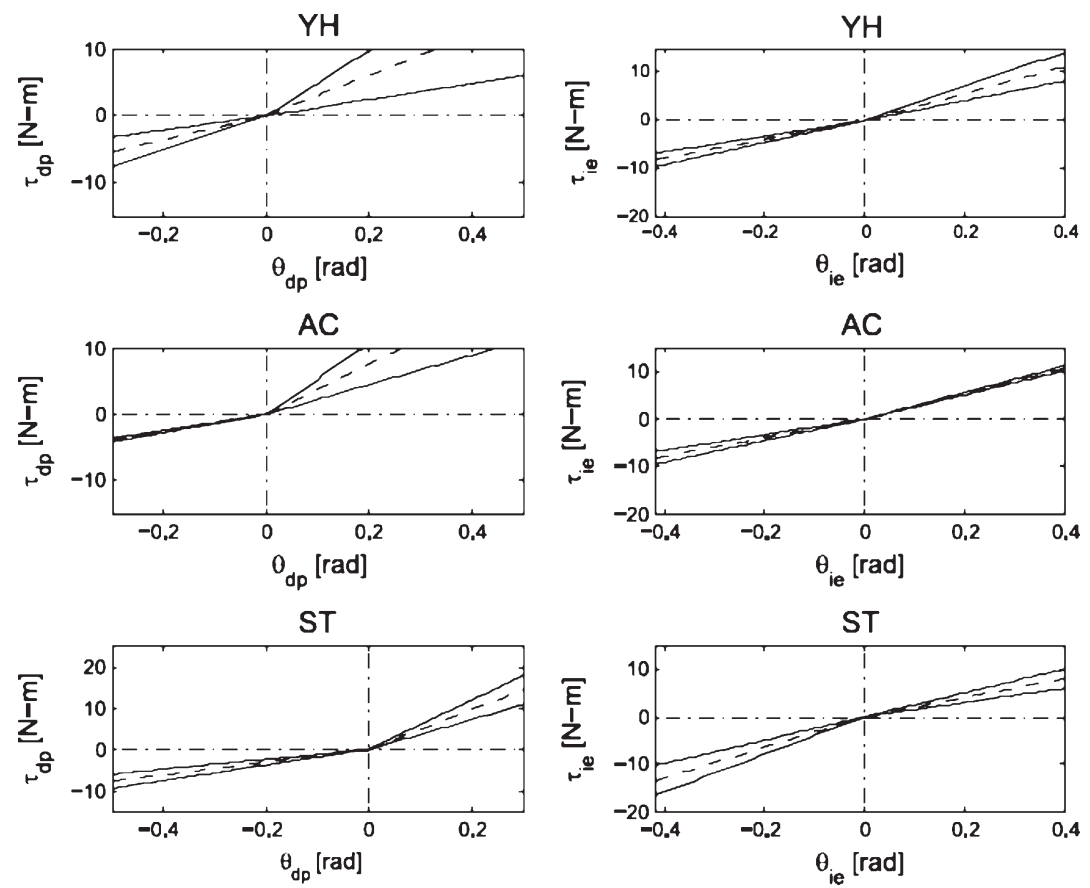

Fig. 3. Least-squares linear regressors showing the mean $\pm \mathrm{SD}$ of the slope (torque vs. ankle angle) in different directions of stretch (left columnsagittal plane, right column-frontal plane) for (top panel) healthy young (YH), (middle panel) age-matched older controls (AC), and (bottom panel) stroke subjects (ST). By convention, angles in dorsiflexion and eversion were considered positive, and those in plantar flexion and inversion were negative. Torque was assigned a polarity consistent with the direction of the movement that it would generate (e.g., dorsiflexion torque was taken as positive). Anatomical neutral was taken as the "zero" position and was determined by positioning the foot on the ground at $90^{\circ}$ with respect to the long axis of the leg. (Adapted from Roy et al., J Neurophysiol 2011).
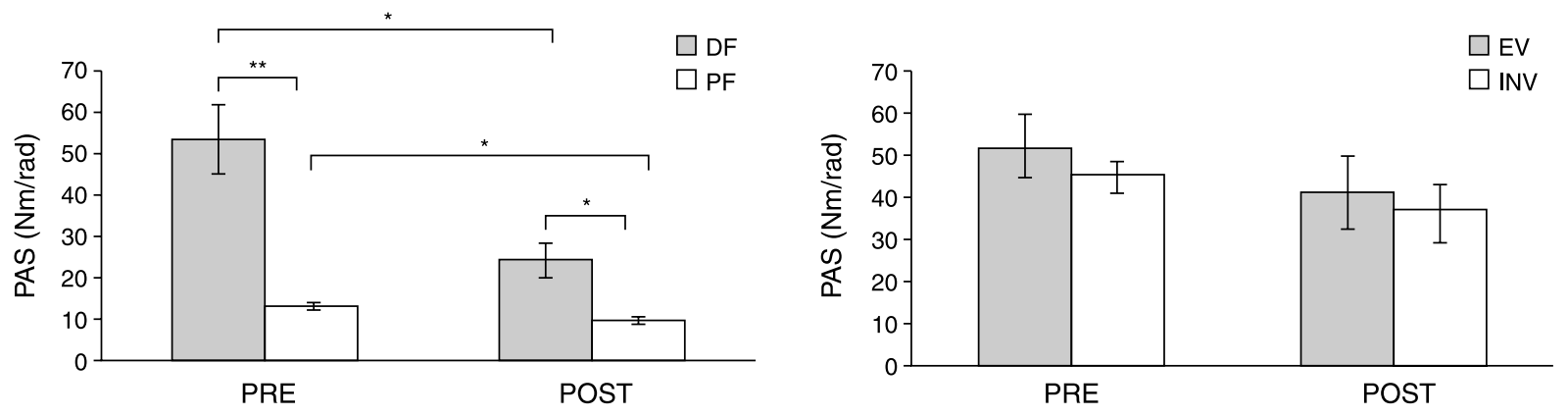

Fig. 4. Changes in PAS before (PRE) and after training (POST): (left panel) Sagittal plane; (right panel) Frontal plane. Unlike the sagittal plane PAS, the frontal plane PAS was not anisotropic at either time points. (Adapted from Roy et al., J Rehabil Res Dev 2013). $\left({ }^{*} p<0.05 ; * * p<0.01\right.$ ).

support stance of unassisted overground gait elicited by robot-assisted ankle training had a significant relationship with baseline PF PAS, while improvements in cadence were strongly linked to baseline EV PAS. These findings suggested that training-induced changes in paretic ankle PAS strongly influence key measures of gait function and constitute novel evidence to link joint-specific impairment to whole-body function.

\section{New directions for seated anklebot training}

The seated visuomotor approach with anklebot has shown promise as a training and clinical assessment platform for persons with chronic hemiparetic stroke. However using the seated format with chronic stroke represents only the first attempts at gauging the device's feasibility for use with stroke generally. It seems likely 
that the greatest impact for this impairment-based application may be with patients in the early phases post-stroke, when the opportunity for whole-body task oriented gait training is often limited in terms of patient stamina and tolerance for high volumes of volitional practice. This is also a period when the biological processes of natural healing and plasticity are potent and may offer a critical window for optimizing the potential for long-term functional recovery. Currently we are investigating seated anklebot training in the early subacute phase, during the course of inpatient treatment in a rehabilitation hospital setting. Preliminary findings in these patients who are severely impaired (floor walking at $<0.20 \mathrm{~m} / \mathrm{s}$ ) have shown that one hour of anklebot therapy is safe and tolerable, while allowing for intensive daily activity using the paretic ankle in over 200 targeted movements (Forrester, Roy, Krywonis, Kehs, Krebs, \& Macko, 2012). These initial results suggest that even relatively few exposures (approximately 10-12 sessions) can improve paretic ankle motor control and some features of gait patterning. Importantly the anklebot sessions have been provided during "off-hours," after completion of usual therapies, which has attracted the interest and support of the therapists on the inpatient stroke unit. A randomized controlled trial is planned to better understand the potential for early timing of this type of LE robotic therapy to impact long term mobility outcomes.

Another potentially valuable direction toward clinical administration of anklebot therapy is to have better understanding of the "state of the learner" as characterized by real-time electrophysiological (e.g., EEG) metrics of brain function (Engelmann, \& Pessoa, 2007; Hosp, Pekanovic, Rioult-Pedotti, \& Luft, 2011; LeDoux, 2003; Lutz, Lachaux, \& Varela, 2002; Salomons, Johnstone, Backonja, \& Davidson, 2004; Schultheiss, 2008; Small, Gitelman, Simmons, Bloise, Parrish, \& Mesulam, 2005). Monitoring brain neurophysiology creates an opportunity to acquire a deeper understanding of central nervous- and motorsystem recovery post-stroke (Fallani et al., 2009; Krakauer, 2006; Mattia, Spanedda, Babiloni, Romigi, \& Marciani, 2003; Mima, Toma, Koshy, \& Hallett, 2001; Nolfe, Cobianchi, Mossuto-Agatiello, \& Giaquinto, 2006; Rijintes, 2006; Strens, Asselman, Pogosyan, loukas, Thompson, \& Brown, 2004;). For example in non-disabled subjects, EEG and other neurophysiologically-based investigations suggest that attentional focus, perceived control and reward elicit brain states that enhance performance and learning (Rijintes, 2006; Schmidt, \& Wrisberg, 2008;

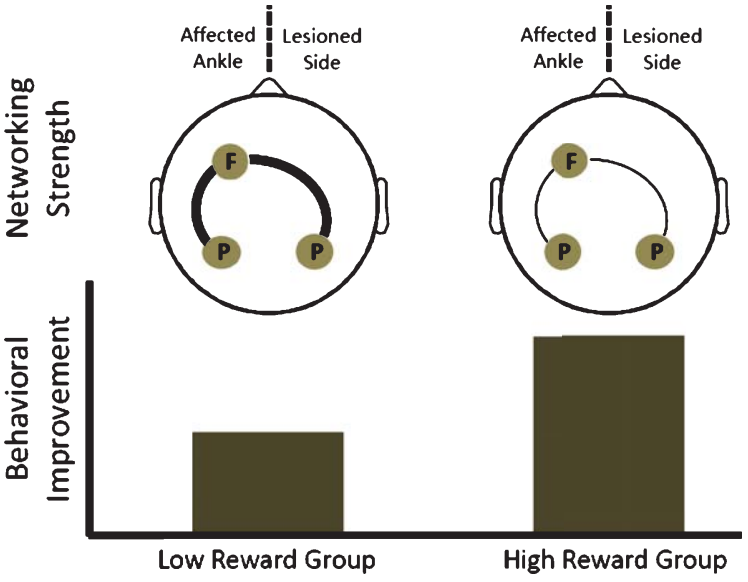

Fig. 5. (Top panel) Strength of networking between the contralesional frontal region $(\mathrm{F})$ and the bihemispheric parietal regions $(\mathrm{P})$ for the two groups (low reward on the left) as a result of the intervention; (bottom panel) relative change in motor performance as compared to before the 3-week ankle robotic training intervention. The high reward group exhibited greater performance gains and reduced networking (seen as thinner connecting lines) as compared to the low reward group

Schultheiss, 2008; Small, Gitelman,Simmons, Bloise, Parrish, \& Mesulam, 2005; Vallerand, 2007). The seated anklebot approach provides a viable platform for modulating task complexity and feedback to promote motor learning while simultaneously monitoring cerebral cortical dynamics.

This potential is illustrated in a comparison of two groups of subjects with chronic stroke $(58.5 \pm 13.5$ yrs; 110 mos. post-stroke; baseline walking speed $8.4 \pm 4.0 \mathrm{~m} / \mathrm{s}$ (range: $0.3-1.3 \mathrm{~m} / \mathrm{s}$ )) who received seated anklebot training for three weeks with high or low reward conditions (Goodman, Macko, Roy, Forrester, 2012). The group that was given overt monetary awards and positive feedback made significant gains in ankle motor control that were associated with more efficient cortical dynamics i.e., reduced networking as compared to low reward controls (Fig. 5). Similar reductions in networking have been linked to motor learning and adaptive behaviors in healthy individuals (Hatfield, Haufler, Hung, \& Spalding, 2004). The addition of this neurophysiologic component helps to guide individualized design of robotic-based interventions and may help predict responders vs. non-responders.

\section{Transitioning to locomotor training with the anklebot}

Consistent with its design, there is also ongoing work aimed at integrating the anklebot module into 
task-specific gait training, both on a treadmill and eventually, over ground in more real-world contexts. One clinical and biomechanical concern is the impact of the asymmetric mass of the robot. We have initiated studies to develop training applications for subjects with stroke. A pilot study investigated the effects of ankle robot mass on gait patterns of chronic stroke survivors to facilitate the design of treadmill-based anklebot training (Khanna, Roy, Rodgers, Krebs, Macko, \& Forrester, 2010). Results indicated that the added inertia and friction of the unpowered anklebot did not significantly alter the paretic and nonparetic step time and stance as percentage of the gait cycle, either in over ground or treadmill conditions. Regardless of loading conditions, the interlimb symmetry as characterized by relative stance durations was greater on the treadmill than over ground. The presence of the unpowered robot loading significantly reduced the nonparetic knee peak flexion on the treadmill and paretic peak dorsiflexion over ground. Overall the results showed that the added mass of the anklebot does not significantly alter the hemiparetic gait pattern.

To begin using the anklebot as a locomotor training stimulus, a basic algorithm was developed that utilizes footswitches to detect the timing of key events in the gait cycle (e.g., paretic heel strike, heel off and toe off) (Fig. 6). This makes it possible to link the level of robotic assistance to specific functional deficits (e.g., foot drop) relative to gait cycle. This method enables the clinician to modify the timing and intensity levels of robotic assistance "on the fly." Moreover, this approach allows more gradual delivery of torques in contrast to a "bang-bang" approach that could destabilize gait. Importantly the rate of torque development to achieve the specified position can be defined to control the intensity of the robotic stimulus, not only adding assistive torque but also giving properly timed proprioceptive cues to enhance volitional push-off and to compensate for possible foot drop in swing. For example it possible to deliver PF torque during late stance into early swing phase, dorsiflex the ankle to assist foot clearance in midswing and if needed, orient the foot for proper landing during late swing. Another key advantage is the ability to adapt to the stride-to-stride variability that is inherent to hemiparetic gait. These technical advances have enabled the integration of anklebot-assisted treadmill based gait training. This work is expected to advance toward more complex adaptive controller models that not only incorporate real time performance data through intrinsic robotic measures, but also from additional inputs that convey the attentional and motivational status (e.g., EEG, rate of perceived exertion) of the patient.

\section{Translating the sub-task control approach into clinical practice}

The event-triggered control scheme enables the anklebot to deliver torques at discrete instants during

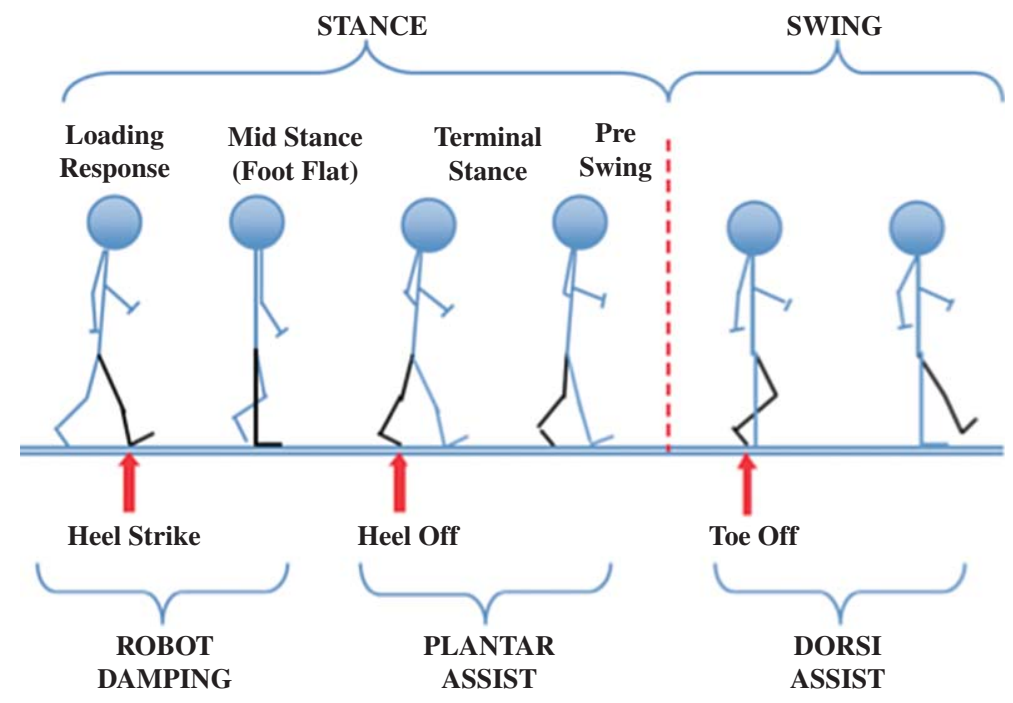

Fig. 6. Timing of anklebot torques can be differentially linked to specific sub-tasks within the gait cycle, and may be adjusted according to patient deficits. The diagram shows three sub-events that are relevant to ankle function throughout the gait cycle. (Adapted from Roy et al., Proc. IEEE ICRA, 2013). 


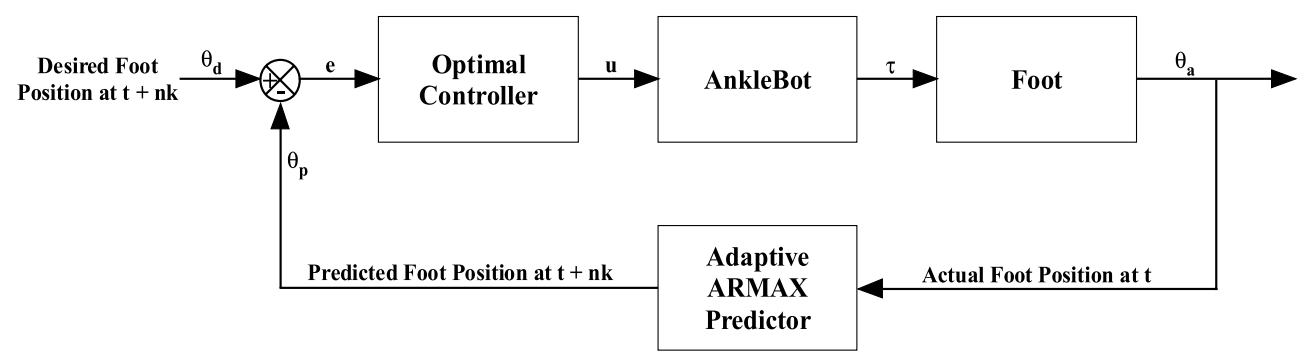

Fig. 7. IM-based feedback control system incorporating an optimal controller determining the optimal anklebot torques and an adaptive ARMAX predictor to predict ankle position via least squares estimation.

the gait cycle e.g., heel strike, heel off, and toe off. Currently, the amounts of torques provided at these events can be manually adjusted by the therapist based on visual observation of the patient's performance. This arrangement permits tailoring the degree assistance provided by the anklebot to match a patient's deficit severity profile, but it is limited in that the process of observing and adjusting assistive torques is too slow to be completed within each individual stride. At best the therapist can observe a number of strides, make an adjustment that affects a subsequent group of strides, and then repeat the observation/adjustment process. Our current capacity with the gait event triggered control algorithm does not automatically update the commanded ankle trajectory (measured by PF and DF angles) across strides. This, interestingly, is the same problem the central nervous system (CNS) must overcome in controlling body and limb movements, which are often times too rapid to be guided by much slower sensory feedback signals. (Here the role of the sensory feedback system is assumed by the therapist.) In part to address this, the concept of the Internal Model (IM) arose (Shadmehr, \& Wise, 2005), which postulates that mobility and balance are controlled by highly adaptable neural structures (Kawato, \& Wolpert, 1998) that represent the kinematics and dynamics of the body and its parts, and the forces and constraints imposed upon them by the external environment. An IM incorporates a predictive element that enables the CNS to predict the consequence of a movement command before the movement is completed, thus allowing in-course corrections to be made. It also incorporates an adaptive element, enabling the predictor to update itself to reflect changes in either the neuromuscular system or the external environment, in order to improve the accuracy of its predictions.

To address these limitations a next step is to develop a predictive/adaptive IM-based controller that will monitor the position of the foot continuously throughout the gait cycle and apply just the amount of torque necessary for adequate forward propulsion during mid- to terminal stance, assistance during mid-swing, and orientation of the foot just prior to landing, much like a therapist, but without the delays inherent in human intervention. As a first pass an adaptive ARMAX (auto-regressive, moving average, exogenous) predictor (Ljung, 1999) is now under development for testing as an optimal controller to determine the appropriate levels of in-course correction (Fig. 7).

The ARMAX predictor is an input-output model and makes no assumptions about the structure of the underlying neuro-physiological processes involved in ankle control.

Moving forward, we will evaluate the Adaptive ARMAX predictor with a Kalman filter (Kalman, 1960). The Kalman filter incorporates a biomechanical model of the ankle dynamics, improving predictive capability and bringing the overall controller development a step closer to representing the actual underlying physiological processes involved in ankle control. Potentially, this may be extended to multiple robotic modules for autonomous multi-segmental control of the lower limb.

\section{Summary}

Early testing of the anklebot has lent support to the idea of using a joint-specific modular approach as a therapeutic modality for hemiparetic stroke. Use of the seated approach has answered initial questions about the potential for augmenting traditional task-specific therapies, and may prove to be a valuable enhancement of early interventions by providing a platform to address underlying impairments in the realm of motor control. Additional work is needed to establish the optimal timing for modular robotics treatment across the spectrum of motor recovery after stroke, beginning with the inpatient rehabilitation hospital setting through 
the outpatient phase of clinical follow-up care. The modular anklebot offers a means to probe the effectiveness of early intensive robotic training for promoting neural plasticity associated with motor learning and whether this will increase the prospects for long term improvements in mobility and balance functions. The integration of the anklebot into actual task-oriented gait training is also evolving toward giving clinicians the ability to focus on specific paretic side deficits, such as foot drop, improper ankle-foot orientation at foot strike, and/or weak propulsion in late stance. In the future we anticipate the real-time integration of EEG-monitored cognitive workload and cortical dynamics with robotderived measures of motor control to sufficiently inform the inputs to the anklebot adaptive controllers to cross a functionally meaningful threshold in the development of more effective robotics-based neurorehabilitation.

\section{Acknowledgments}

The author(s) disclose receipt of the following financial support for the research and/or authorship of this article: This work was funded by the Department of Veterans Affairs Rehabilitation Research and Development Service (VA RR\&D) "Center of Excellence on Task-Oriented Exercise and Robotics in Neurological Diseases," (B3688R); VA RR\&D Merit Pilot Award (A7461P); NIA Claude D. Pepper Older Americans Independence Center (P30-AG028747). The authors acknowledge the Geriatric Research, Education and Clinical Center (GRECC).

\section{Declaration of conflicting interests}

The author(s) declared a potential conflict of interest (e.g. a financial relationship with the commercial organizations or products discussed in this article) as follows: Dr. H. I. Krebs is a co-inventor in the MIT patents for the robotic devices. He holds equity positions in Interactive Motion Technologies, Inc., the company that manufactures this type of technology under license to MIT.

\section{References}

Barbeau, H., \& Visintin, M. (2003). Optimal outcomes obtained with body-weight support combined with treadmill training in stroke subjects. Archives of Physical Medicine and Rehabilitation, 84(10), 1458-1465.
Bowden, M. G., Balasubramanian, C. K., Neptune, R. R., \& Kautz, S. A. (2006). Anterior-posterior ground reaction forces as a measure of paretic leg contribution in hemiparetic walking. Stroke, 37(3), 872-876.

Boyce, B. A., Coker, C. A., \& Bunker, L. K. (2006). Implications for variability of practice from pedagogy and motor learning perspectives: Finding a common ground. Quest, 58(3), 330-343.

Burgar, C. G., Lum, P. S., Scremin, A. M., Garber, S. L., Van der Loos, H. L., Kenney, D., \& Shor, P. (2011). Robot-assisted upperlimb therapy in acute rehabilitation setting following stroke: Department of Veterans Affairs multisite clinical trial. Journal of Rehabilitation Research and Development, 48, 445-458.

Colombo, G., Joerg, M., Schreier, R., \& Dietz, V. (2000). Treadmill training of paraplegic patients using a robotic orthosis. Journal of Rehabilitation Research and Development, 37(6), 693-700.

Colombo, G., Joerg, M., Schreier, R., \& Dietz, V. (2000). Treadmill training of paraplegic patients using a robotic orthosis. Journal of Rehabilitation Research and Development, 37(6), 693-700.

De Vico Fallani, F., Astolfi, L., Cincotti, F., Mattia, D., la Rocca, D., Maksuti, E., \& Nagy, Z.(2009). Evaluation of the brain network organization from EEG signals: A preliminary evidence in stroke patient. The Anatomical Record, 292(12), 2023-2031.

Dennis, M. S., Lo, K. M., McDowall, M., \& West, T. (2002). Fractures after stroke: Frequency, types, and associations. Stroke, 33(3), 728-734.

Duncan, P. W., Sullivan, K. J., Behrman, A. L., Azen, S. P., Wu, S. S., Nadeau, S. E., \& Hayden, S. K.(2011). Body-weight-supported treadmill rehabilitation after stroke. New England Journal of Medicine, 364(21), 2026-2036.

Engelmann, J. B., \& Pessoa, L. (2007). Motivation sharpens exogenous spatial attention. Emotion, 7(3), 668-674.

Forrester, L. W., Roy, A., Krebs, H. I., \& Macko, R. F. (2011). Performance-based ankle robotics training improves hemiparetic gait after stroke: A preliminary report. Neurorehabilitation and Neural Repair, 25, 369-377.

Forrester, L., Roy, A., Krywonis, A., Krebs, H. I., \& Macko, R. (2012). Poster 90 feasibility for using ankle robotics in the acute phase of stroke: A controlled pilot study. Archives of Physical Medicine and Rehabilitation, 93(10), e40.

Forster, A., \& Young, J. (1995). Incidence and consequences of falls due to stroke: A systematic inquiry. British Medical Journal, 311(6997), 83-86.

Goodman, R. N., Forrester, L., Macko, R., \& Roy, A. (2012). Poster 29 cortical and biomechanical dynamics of lower extremity robotics training at different levels of motivational incentive. Archives of Physical Medicine and Rehabilitation, 93(10), e22.

Gordon, D., Robertson, E., \& Winter, D. A. (1980). Mechanical energy generation, absorption and transfer amongst segments during walking. Journal of Biomechanics, 13(10), 845-854.

Grillner, S. (1975). Locomotion in vertebrates: Central mechanisms and reflex interaction. Physiological Reviews, 55(2), 247-304.

Grillner, S., \& Wallen, P. (1985). Central pattern generators for locomotion, with special reference to vertebrates. Annual Review of Neuroscience, 8(1), 233-261.

Halsband, U., \& Lange, R. K. (2006). Motor learning in man: A review of functional and clinical studies. Journal of Physiology, Paris, 99(4-6), 414-424.

Hassid, E., Rose, D., Commisarow, J., Guttry, M., \& Dobkin, B. H. (1997). Improved gait symmetry in hemiparetic stroke patients induced during body weight-supported treadmill stepping. Neurorehabilitation and Neural Repair, 11(1), 21-26. 
Hatfield, B. D., Haufler, A. J., Hung, T. M., \& Spalding, T. W. (2004). Electroencephalographic studies of skilled psychomotor performance. Journal of Clinical Neurophysiology, 21(3), 144-156.

Hesse, S., \& Uhlenbrock, D. (2000). A mechanized gait trainer for restoration of gait. Journal of Rehabilitation Research and Development, 37(6), 701-708.

Hesse, S., Bertelt, C., Jahnke, M. T., Schaffrin, A., Baake, P., Malezic, M., \& Mauritz, K. H. (1995). Treadmill training with partial body weight support compared with physiotherapy in nonambulatory hemiparetic patients. Stroke, 26(6), 976-981.

Hesse, S., Bertelt, C., Schaffrin, A., Malezic, M., \& Mauritz, K. H. (1994). Restoration of gait in nonambulatory hemiparetic patients by treadmill training with partial body-weight support. Archives of Physical Medicine and Rehabilitation, 75(10), 1087.

Hornby, T. G., Campbell, D. D., Kahn, J. H., Demott, T., Moore, J. L., \& Roth, H. R. (2008). Enhanced gait-related improvements after therapist-versus robotic-assisted locomotor training in subjects with chronic stroke: A randomized controlled study. Stroke, 39(6), 1786-1792.

Hosp, J. A., Pekanovic, A., Rioult-Pedotti, M. S., \& Luft, A. R. (2011). Dopaminergic projections from midbrain to primary motor cortex mediate motor skill learning. The Journal of Neuroscience, 31(7), 2481-2487.

Ichinose, W. E., Reinkensmeyer, D. J., Aoyagi, D., Lin, J. T., Ngai, K., Edgerton, V. R., \& Bobrow, J. E. (2003). A robotic device for measuring and controlling pelvic motion during locomotor rehabilitation. In Engineering in Medicine and Biology Society, 2003. Proceedings of the 25th Annual International Conference of the IEEE (Vol. 2, pp. 1690-1693). IEEE.

Kalman, R. E. (1960). A new approach to linear filtering and prediction problems. Journal of Basic Engineering, 82(1), 35-45.

Kanis, J., Oden, A., \& Johnell, O. (2001). Acute and long-term increase in fracture risk after hospitalization for stroke. Stroke, 32(3), 702-706

Kawato, M., \& Wolpert, D. (1998). Internal models for motor control. Sensory Guidance of Movement, 218, 291-307.

Khanna, I., Roy, A., Rodgers, M. M., Krebs, H. I., Macko, R. M., \& Forrester, L. W. (2010). Effects of unilateral robotic limb loading on gait characteristics in subjects with chronic stroke. Journal of NeuroEngineering and Rehabilitation, 7(1), 1-8.

Krakauer, J. W. (2006). Motor learning: Its relevance to stroke recovery and neurorehabilitation. Current Opinion in Neurology, 19(1), 84-90.

Krebs, H. I., Hogan, N., Aisen, M. L., \& Volpe, B. T. (1998). Robotaided neurorehabilitation. IEEE Transactions on Rehabilitation Engineering, 6(1), 75-87.

Krebs, H. I., Volpe, B. T., Lynch, D., \& Hogan, N. (2005). Stroke rehabilitation: An argument in favor of a robotic gym. Paper presented at the Proceedings of the 9th International Conference on Rehabilitation Robotics (USA), Chicago, USA, pp. 219-222.

Krebs, H. I., Volpe, B. T., Williams, D., Celestino, J., Charles, S. K., Lynch, D., \& Hogan, N. (2007). Robot-aided neurorehabilitation: A robot for wrist rehabilitation. IEEE Transactions on Neural Systems and Rehabilitation Engineering, 15(3), 327-335.

LeDoux, J.E. (2003). Synaptic self: How our brains become who we are. Penguin.

Ljung, L. (1999). System Identification. John Wiley \& Sons, Inc.

Lo, A. C., Guarino, P. D., Richards, L. G., Haselkorn, J. K., Wittenberg, G. F., Federman, D. G., \& Peduzzi, P. (2010). Robot-assisted therapy for long-term upper-limb impairment after stroke. New England Journal of Medicine, 362(19), 1772-1783.
Luft, A. R., Macko, R. F., Forrester, L. W., Villagra, F., Ivey, F., Sorkin, J. D., \& Hanley, D. F. (2008). Treadmill exercise activates subcortical neural networks and improves walking after stroke: A randomized controlled trial. Stroke, 39(12), 3341-3350.

Lum, P. S., Burgar, C. G., Kenney, D. E., \& Van der Loos, H. F. (1999). Quantification of force abnormalities during passive and active-assisted upper-limb reaching movements in post-stroke hemiparesis. IEEE Transactions on Biomedical Engineering, 46(6), 652-662.

Lutz, A., Lachaux, J. P., Martinerie, J., \& Varela, F. J. (2002). Guiding the study of brain dynamics by using first-person data: Synchrony patterns correlate with ongoing conscious states during a simple visual task. Proceedings of the National Academy of Sciences, 99(3), 1586-1591.

Macko, R. F., Ivey, F. M., Forrester, L. W., Hanley, D., Sorkin, J. D., Katzel, L. I., \& Goldberg, A. P. (2005). Treadmill exercise rehabilitation improves ambulatory function and cardiovascular fitness in patients with chronic stroke: A randomized, controlled trial. Stroke, 36(10), 2206-2211.

Mattia, D., Spanedda, F., Babiloni, F., Romigi, A., \& Marciani, M. G. (2003). Quantitative EEG patterns following unilateral stroke: A study in chronic stage. International Journal of Neuroscience, 113(4), 465-482.

Mehrholz, J., Werner, C., Kugler, J., \& Pohl, M. (2007). Electromechanical-assisted training for walking after stroke. Cochrane Database Sys Rev, (4), CD006185. DOI: 10.1002/14651858.CD006185.pub2

Mima, T., Toma, K., Koshy, B., \& Hallett, M. (2001). Coherence between cortical and muscular activities after subcortical stroke. Stroke, 32(11), 2597-2601.

Mirelman, A., Bonato, P., \& Deutsch, J. E. (2009). Effects of training with a robot-virtual reality system compared with a robot alone on the gait of individuals after stroke. Stroke, 40(1), 169-174.

Nashner, L. M. (1977). Fixed patterns of rapid postural responses among leg muscles during stance. Experimental Brain Research, 30(1), 13-24.

Nolfe, G., Cobianchi, A., Mossuto-Agatiello, L., \& Giaquinto, S. (2006). The role of P300 in the recovery of post-stroke global aphasia. European Journal of Neurology, 13(4), 377-384.

Olney, S. J., Griffin, M. P., Monga, T. N., \& McBride, I. D. (1991). Work and power in gait of stroke patients. Archives of Physical Medicine and Rehabilitation, 72(5), 309.

Pessoa, L. (2009). How do emotion and motivation direct executive control? Trends in Cognitive Sciences, 13(4), 160-166.

Ramnemark, A., Nyberg, L., Borssen, B., Olsson, T., \& Gustafson, Y. (1998). Fractures after stroke. Osteoporosis International, 8(1), 92-95.

Reinkensmeyer, D. J., Dewald, J. P., \& Rymer, W. Z. (1999). Guidance-based quantification of arm impairment following brain injury: A pilot study. IEEE Transactions on Rehabilitation Engineering, 7(1), 1-11.

Reisman, D. S., Wityk, R., Silver, K., \& Bastian, A. J. (2007). Locomotor adaptation on a split-belt treadmill can improve walking symmetry post-stroke. Brain, 130(7), 1861-1872.

Reisman, D. S., Wityk, R., Silver, K., \& Bastian, A. J. (2009). Split-belt treadmill adaptation transfers to overground walking in persons poststroke. Neurorehabilitation and Neural Repair, 23(7), 735-744.

Rijntjes, M. (2006). Mechanisms of recovery in stroke patients with hemiparesis or aphasia: New insights, old questions and the meaning of therapies. Current Opinion in Neurology, 19(1), 76-83. 
Roy, A., Forrester, L. W., \& Macko, R. F. (2011). Short-term ankle motor learning with ankle robotics training in chronic hemiparetic stroke. Journal of Rehabilitation Research and Development, 48(4), 417-429.

Roy, A., Forrester, L. W., Macko, R. F., \& Krebs, H. I. (2013). Changes in passive ankle stiffness and its effects on gait function in people with chronic stroke. J Rehabil Res Dev, 50(4), XX-XX. DOI: 10.1682/JRRD.2011.10.0206

Roy, A., Krebs, H. I., Barton, J. E., Macko, R. F., \& Forrester, L. W. (2013). Anklebot-assisted locomotor training after stroke: A novel deficit-adjusted approach. In: Proc. IEEE Int. Conf. on Robotics and Automation (ICRA), Karlsruhe, Germany, 21672174.

Roy, A., Krebs, H. I., Bever, C. T., Forrester, L. W., Macko, R. F., \& Hogan, N. (2011). Measurement of passive ankle stiffness in subjects with chronic hemiparesis using a novel ankle robot. Journal of Neurophysiology, 105(5), 2132-2149.

Roy, A., Krebs, H. I., Williams, D. J., Bever, C. T., Forrester, L. W., Macko, R. M., \& Hogan, N. (2009). Robot-aided neurorehabilitation: A novel robot for ankle rehabilitation. Robotics, IEEE Transactions on, 25(3), 569-582.

Salomons, T. V., Johnstone, T., Backonja, M. M., \& Davidson, R. J. (2004). Perceived controllability modulates the neural response to pain. The Journal of Neuroscience, 24(32), 7199-7203.

Schmidt, H., Sorowka, D., Hesse, S., \& Bernhardt, R. (2003). Development of a robotic walking simulator for gait rehabilitation. Biomedizinische Technik, 48(10), 281-286.
Schmidt, H., Werner, C., Bernhardt, R., Hesse, S., \& Krüger, J. (2007). Gait rehabilitation machines based on programmable footplates. Journal of Neuroengineering and Rehabilitation, 4(1), 2.

Schmidt, R. A., \& Wrisberg, C. A. (2008). Motor learning and performance: A situation-based learning approach. Human Kinetics Publishers.

Schultheiss, O. C., Wirth, M. M., Waugh, C. E., Stanton, S. J., Meier, E. A., \& Reuter-Lorenz, P. (2008). Exploring the motivational brain: Effects of implicit power motivation on brain activation in response to facial expressions of emotion. Social Cognitive and Affective Neuroscience, 3(4), 333-343.

Shadmehr, R., \& Wise, S. P. (2005). The Computational Neurobiology of Reaching and Pointing: A Foundation for Motor Learning. The MIT press.

Small, D. M., Gitelman, D., Simmons, K., Bloise, S. M., Parrish, T., \& Mesulam, M. M. (2005). Monetary incentives enhance processing in brain regions mediating top-down control of attention. Cerebral Cortex, 15(12), 1855-1865.

Strens, L. H. A., Asselman, P., Pogosyan, A., Loukas, C., Thompson, A. J., \& Brown, P. (2004). Corticocortical coupling in chronic stroke Its relevance to recovery. Neurology, 63(3), 475-484.

Vallerand, R. J. (2007). Intrinsic and Extrinsic Motivation in Sport and Physical Activity: A Review and a Look at the Future. Handbook of Sport Psychology, Third Edition, 59-83. 62.

Westlake, K. P., \& Patten, C. (2009). Pilot study of Lokomat versus manual-assisted treadmill training for locomotor recovery poststroke. J NeuroEng Rehabil, 6, 18. DOI: 10.1186/1743-0003-6-18 